\title{
BMJ Open Interventions and practice models for improving health and psychosocial outcomes of children and young people in out-of-home care: protocol for a systematic review
}

\author{
Emma Galvin, ${ }^{1}$ Renée O'donnell, ${ }^{1}$ Helen Skouteris, ${ }^{1,2}$ Nick Halfpenny, ${ }^{3}$ \\ Aya Mousa $^{1}$
}

To cite: Galvin E, O'donnell R, Skouteris $\mathrm{H}$, et al. Interventions and practice models for improving health and psychosocial outcomes of children and young people in out-of-home care: protocol for a systematic review. BMJ Open 2019;9:e031362. doi:10.1136/ bmjopen-2019-031362

- Prepublication history and additional material for this paper are available online. To view these files, please visit the journal online (http://dx.doi org/10.1136/bmjopen-2019031362).

Received 30 April 2019 Revised 19 July 2019 Accepted 25 July 2019

\section{Check for updates}

C Author(s) (or their employer(s)) 2019. Re-use permitted under CC BY-NC. No commercial re-use. See rights and permissions. Published by BMJ.

${ }^{1}$ School of Public Health and Preventive Medicine, Monash Centre for Health Research and Implementation, Monash University, Melbourne, Victoria, Australia

${ }^{2}$ School of Business, University of Warwick, Coventry, UK

${ }^{3}$ Policy and Research, MacKillop Family Services, Melbourne, Victoria, Australia

Correspondence to Professor Helen Skouteris; helen.skouteris@monash.edu

\section{ABSTRACT}

Introduction Children and young people placed in out-of-home care $(\mathrm{O} \mathrm{oHC})$ are often affected by a history of trauma and adverse childhood experiences. Trauma in early childhood can impact on children's health and psychosocial development, whereas early interventions can improve children's development and placement stability. Although several interventions and practice models have been developed to improve health and psychosocial outcomes for children and young people in $\mathrm{OoHC}$, there remains a lack of rigorous research examining the impact of these interventions in $\mathrm{OOHC}$ settings, as there are no systematic reviews examining the impact these interventions and practice models have on the children and young people they serve. We aim to conduct a comprehensive systematic review to examine the effectiveness of interventions and practice models for improving health and psychosocial outcomes in children and young people living in $\mathrm{OoHC}$ and to identify relevant knowledge gaps.

Methods and analysis Major electronic databases including Medline, Medline in-process and other nonindexed citations, Embase, Cumulative Index to Nursing and Allied Health Literature, Psyclnfo, Sociological Abstracts and all Evidence-Based Medicine Reviews incorporating: Cochrane Database of Systematic Reviews, American College of PhysiciansJournal Club, Database of Abstracts of Reviews of Effects,Cochrane Central Register of Controlled Trials, CochraneMethodology Register, Health Technology Assessment and National Health Service Economic Evaluation Database, will be systematically searched for any studies published between 2008 and 2018 of interventions and practice models developed to improve health and psychosocial outcomes for children and young people in OoHC. Two independent reviewers will assess titles and abstracts for eligibility according to prespecified selection criteria and will perform data extraction and quality appraisal. Meta-analyses and/or metaregression will be conducted where appropriate. Ethics and dissemination This study will not collect primary data and formal ethical approval is therefore not required. Findings from this systematic review will be disseminated in a peer-reviewed publication and conference presentations.
Strengths and limitations of this study

- The current study employs rigorous international gold-standard methodology and a comprehensive search strategy.

- Limitations of this study include the potential for publication bias since the systematic review will include only published data;

- This study includes the potential that studies may be too heterogeneous to obtain combined effect estimates.

PROSPERO registration number CRD42019115082.

\section{INTRODUCTION}

Children and young people in out-of-home care $(\mathrm{OoHC})$ are some of the most vulnerable groups in society, often having experienced substantial harm, abuse or neglect. ${ }^{12}$ OoHC refers to the short-term or long-term care of children and young people up to 18 or 21 years of age (depending on country) who are unable to live with their families due to child protection orders and/or as a result of parents being unable to provide adequate care or protection. ${ }^{3}$ In Australia, 47915 children $<18$ years lived in OoHC in 2017, a rate of 8.7 per 1000 children, reflecting an increase from 46448 and 40549 children in 2016 and 2013, respectively. ${ }^{2}{ }^{4}$ These rising rates are concerning, since children and young people placed in OoHC are often characterised as having severe cognitive, emotional, behavioural and social problems, ${ }^{15}$ coupled with complex histories of maltreatment and neglect. ${ }^{56}$ This history of trauma is believed to have short-term and long-term effects on brain development, from childhood through to adulthood, and 
often culminates into complex behavioural, psychological and social challenges. ${ }^{56}$ Children and young people in OoHC report poorer outcomes across a number of health and well-being indicators compared with those who remain with their biological family. ${ }^{7}$ Since children and young people usually enter care having experienced trauma and neglect, deviant behaviour and mental health problems are particularly prevalent among children and young people in OoHC, and this appears to be associated with both age at first placement and type of care. ${ }^{8}$ A 2006 study found that up to $60 \%$ of children and young people in OoHC have a current mental health diagnosis including depression, attachment and conduct disorders, and attention deficit hyperactivity disorders, ${ }^{9}$ and those placed in residential care tend to have higher rates compared with those in foster care, whereas individuals in kinship care report the fewest rate of mental health disorders. ${ }^{810}$ Children and young people in OoHC also report a significantly higher incidence of substance abuse, suicide ideation and suicide attempt, ${ }^{1-13}$ as well as attachment difficulties, problematic sexual behaviour, eating disorders, delinquent behaviour and reduced educational attainment compared with children and young people residing with their biological families. ${ }^{814}$ The poor outcomes that children and young people in OoHC face, some of which are mentioned above, play a significant role in the complexity of their overall health, making it more challenging to identify their health needs and develop appropriate health management plans. Evidently, children and young people in OoHC require more intensive intervention and support, as we are dealing with complex, multifaceted issues, that require a number of strategies that can effectively support their health and well-being.

In light of the poor health and psychosocial outcomes experienced by children and young people living in OoHC, effective and sustainable interventions for improving these outcomes are urgently needed. Over the last few years, a number of practice models and interventions have been developed with the aim of directly addressing the impact of trauma on health and psychosocial outcomes for children and young people in OoHC. ${ }^{15}$ Some of these models, such as the Sanctuary Model, Therapeutic Residential Care and Treatment Foster Care are shifting towards needs-based care and incorporating a trauma-informed, therapeutic care approach within the OoHC placements. ${ }^{16}$ In Australia, the UK and the USA, it is expected that children and young people entering OoHC have the appropriate health assessments (statutory) and that healthcare records and management plans are in place. ${ }^{17-20}$ Unfortunately, this process is not always met, and even when a child is provided with a health management plan, their needs may not be incorporated and the plan may not be followed. ${ }^{18}$ Collecting the necessary information can be difficult as health professionals must rely on parents to provide medical histories, explain health and behavioural concerns and consent to the assessment and treatment of their child. ${ }^{18}$ Despite carers' and case managers' best efforts to provide this information, the high percentage of placement breakdowns and constant change in caregivers and service providers create gaps in information pertaining to the individuals' social or family circumstances and medical and mental health, and there is risk of this information being lost. ${ }^{6}$

Most interventions have also not been properly evaluated, and there remains a lack of rigorous research examining the impact of these interventions in improving health and/or psychosocial outcomes for children and young people in OoHC. ${ }^{21}$ Indeed, a recent systematic review investigating the empirical evidence of trauma-informed, organisation-wide models implemented in residential OoHC settings identified three models including The Sanctuary Model, Children and Residential Experiences programme and the Attachment Regulation and Competency framework, and concluded that the evidence base is limited, making it difficult to accurately evaluate outcomes of trauma-informed models. ${ }^{22}$ Recent studies have outlined the health and psychosocial needs of children and young people in OoHC and the interventions and practice models that have been designed to meet these needs; however, to date, very little research has been focused on evaluating the effectiveness of these interventions. ${ }^{15}$ To our knowledge, no previous systematic reviews have examined interventions or practice models designed to respond to the physical and psychosocial health needs of children or young people in residential, foster and kinship settings of OoHC. To develop effective, evidence-based interventions in OoHC, we first need to understand which interventions and practice models work and how their structures and processes can be implemented and sustained in practice. Otherwise, the cycle of disadvantage and poor health outcomes will not be broken, and children and young people in OoHC will remain at increased risk of adverse health and psychosocial outcomes. To this end, we aim to conduct a comprehensive systematic review which will: (1) assess the effectiveness of interventions and practice models for improving the health and psychosocial outcomes of children and young people living in OoHC (all types of placements); (2) examine whether a particular intervention or practice model is more effective than another and (3) delineate which components of these interventions are associated with the greatest improvement in outcomes for these children and young people.

\section{Systematic review questions}

- Are certain interventions or practice models effective in improving health and/or psychosocial outcomes for children and young people in OoHC compared with usual care?

- Are certain interventions or practice models more effective than others in improving health and/or psychosocial outcomes for children and young people in $\mathrm{OoHC}$ ?

- Which elements are critical in determining the success of interventions, and for whom? 


\begin{tabular}{|c|c|c|c|c|c|c|}
\hline & Participants (P) & Intervention (I) & Comparison (C) & Outcomes (0) & Study type & Limits \\
\hline $\begin{array}{l}\text { Inclusion } \\
\text { criteria }\end{array}$ & $\begin{array}{l}\text { Children } \\
0-21 \text { years of } \\
\text { age and living in } \\
\text { OoHC } \\
\text { School aged } \\
\text { Youth } \\
\text { Adolescents } \\
\text { Children } \\
\text { Infants }\end{array}$ & $\begin{array}{l}\text { Any intervention } \\
\text { (eg, treatment } \\
\text { foster care; } \\
\text { therapeutic } \\
\text { residential care, } \\
\text { sanctuary model, } \\
\text { etc) delivered in } \\
\text { an OoHC setting } \\
\text { (eg, foster care, } \\
\text { kinship care, } \\
\text { residential care). }\end{array}$ & $\begin{array}{l}\text { No intervention; } \\
\text { usual care; other } \\
\text { interventions in } \\
\text { OoHC; } \\
\text { children who } \\
\text { remain with their } \\
\text { biological/ foster } \\
\text { families. }\end{array}$ & $\begin{array}{l}\text { All health and } \\
\text { psychosocial } \\
\text { outcomes including } \\
\text { but not limited to: } \\
\text { intellectual; } \\
\text { behavioural; } \\
\text { psychosocial; } \\
\text { mental; suicidal } \\
\text { ideation; } \\
\text { psychological } \\
\text { functioning; social } \\
\text { skills; emotional; } \\
\text { educational } \\
\text { attainment; } \\
\text { relationships; illicit } \\
\text { drug use; smoking; } \\
\text { alcohol; eating } \\
\text { disorders. }\end{array}$ & $\begin{array}{l}\text { Randomised } \\
\text { controlled trials; } \\
\text { non-randomised or } \\
\text { uncontrolled trials; } \\
\text { systematic reviews; } \\
\text { cohort studies; } \\
\text { cross-sectional; } \\
\text { longitudinal. }\end{array}$ & $\begin{array}{l}\text { English } \\
\text { language only; } \\
\text { peer-reviewed; } \\
\text { published in the } \\
\text { last 10years } \\
\text { (2008-2018). }\end{array}$ \\
\hline $\begin{array}{l}\text { Exclusion } \\
\text { criteria }\end{array}$ & $\begin{array}{l}\text { Adults } \\
>21 \text { years of age }\end{array}$ & $\begin{array}{l}\text { Adoption; } \\
\text { rehabilitation; } \\
\text { orphanages }\end{array}$ & & & $\begin{array}{l}\text { Editorial; } \\
\text { commentary; } \\
\text { narrative review; } \\
\text { expert opinion }\end{array}$ & $\begin{array}{l}\text { Literature } \\
\text { published } \\
\text { before 2008; } \\
\text { Languages } \\
\text { other than } \\
\text { English. }\end{array}$ \\
\hline
\end{tabular}

OoHC, out-of-home care; PICO, Population, Intervention, Comparison, Outcomes.

\section{METHODS AND ANALYSIS}

This systematic review uses rigorous international gold standard methodology $y^{23}$ and conforms to the reporting standards of the Preferred Reporting Items for Systematic Reviews and Meta-analyses ${ }^{25}$ (PRISMA; see online supplementary file).

\section{Selection criteria}

As outlined in table 1, a Population, Intervention, Comparison, Outcomes framework was established a priori to screen studies and determine their eligibility for inclusion in the systematic review. Interventions and practice models developed for reunification have been excluded; however, interventions and practice models that incorporate participants who transitioned into a different type of care setting are included.

\section{Search strategy}

A systematic search, based on the selection criteria (table 1) and combining medical subject heading terms and text words, was developed using the Ovid platform and translated to other databases as appropriate (see online supplementary file). The search terms are outlined in box 1.

Relevant articles will be sourced through electronic databases including: Medline, Medline in-process and other non-indexed citations, Embase, PsycINFO, Cumulative Index to Nursing and Allied Health Literature, Sociological Abstracts and all Evidence-Based Medicine Reviews incorporating: Cochrane Database of Systematic
Reviews, American College of Physicians Journal Club, Database of Abstracts of Reviews of Effects, Cochrane Central Register of Controlled Trials, Cochrane Methodology Register, Health Technology Assessment, National Health Service Economic Evaluation Database. Bibliographies of relevant studies as well as systematic reviews identified by the search strategy will be screened for identification of additional studies. Where required data are not presented, the corresponding authors of included studies will be contacted to provide deidentified aggregate data for the purpose of meta-analyses if deemed necessary.

\section{Box 1 Sample of search terms used in electronic search}

\section{Concept 1: Out of home care}

Foster care, foster, out of home, kinship, trauma informed, resident*, guardian care, family based care, family centered, home based, child protection, child welfare, non biological care, group home, group house, 'OOHC'

\section{Concept 2: Participants}

Looked after child*, young person, young people, infan*, baby, babies, toddler, preschool*, adolescen*, teen*, minor, youth

\section{Concept 3: Intervention}

Model, outcome, evaluation, framework, theor*, intervention, program*, process $^{\star}$, prevention, treatment, strategy ${ }^{\star}$, therap*, trauma informed, trauma focused, trauma service 


\section{Screening of search results}

Search results will be managed using the Endnote V.X.8.0 reference management software. Two reviewers will assess the titles, abstracts and keywords of every article retrieved by the search strategy according to the selection criteria described in table 1 . Full text of the articles will be retrieved for further assessment if the information provided suggests that the study meets the selection criteria or if there is any doubt regarding eligibility of the article based on the information given in the title and abstract. Where there is more than one article describing the same study and reporting different outcomes, these articles will be combined and considered a single unique study. Articles excluded after full-text assessment will be tabulated with reasons for their exclusion, as per PRISMA guidelines. $^{26}$

\section{Data extraction}

Two independent reviewers will formally screen the included studies against the selection criteria and perform data extraction using a specifically designed data extraction form. Extracted data will include general study characteristics (author, year, country, setting, inclusion/ exclusion criteria), population characteristics (gender and age distribution and other relevant features), intervention and control characteristics (type/model, duration, frequency and so on), outcome assessments (physical and psychosocial outcomes and tools used to assess these) and results (point estimates and measures of variability for continuous outcomes and frequency counts or absolute numbers of episodes or relative measures of risk (risk ratio or ORs with CIs) for dichotomous variables, numbers of participants, intention-to-treat analysis) and any other relevant validity results. Missing data will be obtained from corresponding authors wherever possible, and two reviewers will check all computed data entries for meta-analysis if applicable. Any disagreement will be resolved by discussion to reach a consensus.

\section{Assessment of risk of bias and quality of the evidence}

Methodological quality of included studies will be assessed at the study-level by two independent reviewers using a risk of bias assessment template according to study design. Individual quality items will be investigated using a descriptive component approach which will include assessment of key aspects such as methods of outcome assessment and reporting, statistical analysis components including study power and dealing with confounding, attrition rates and conflicts of interest of authors. Using this process, a risk of bias rating (high, moderate or low) will be assigned to each study.

Quality of the evidence for the effects of interventions in improving health and psychosocial outcomes for children and young people in OoHC will be assessed by two independent reviewers using the Grading of Recommendations, Assessment, Development and Evaluations framework. ${ }^{27}$ This will be used to appraise quality at the outcome level and, where appropriate, will incorporate aspects such as risk of bias, inconsistency, indirectness, imprecision and publication bias. Based on this evaluation, a quality score (high, moderate, low or very low) will be assigned to each outcome. Disagreement will be resolved by discussion to reach consensus.

\section{Data analysis and synthesis}

Data will be presented in summary form and narratively as well as in tables (where possible) to describe the study designs, populations and findings and to address each research question. Data will be summarised statistically using meta-analysis of aggregate effect measures if available and if studies are deemed sufficiently homogeneous to combine. The meta-analysis will be performed on studies in which a baseline and follow-up effect is available (ie, randomised control trial and quasi-experimental) and wherein the same outcome of interest has been reported (ie, anxiety, depression, self-harming behaviour, delinquent behaviour, obesity) along with a change in effect. As the outcomes of interest will likely be assessed using a diverse range of instruments, a random effects model will be estimated accounting for the heterogeneity between the studies. Review Manager V.5 software will be used for meta-analysis, and results will be expressed as relative risks or ORs with 95\% CIs for dichotomous outcomes and weighted mean differences with $95 \%$ CI for continuous outcomes. Statistical homogeneity will be assessed using the $\mathrm{I}^{2}$ test where $\mathrm{I}^{2}$ values over $50 \%$ indicate moderate to high heterogeneity. ${ }^{28}$ Statistical significance will be set at a two-tailed $p<0.05$. For studies with qualitative designs or have insufficient data for pooling, a descriptive analysis will be presented.

\section{Subgroup analysis}

Subgroup analysis, and where appropriate, meta-regression will be performed if possible based on study characteristics and results from the search. Where there is sufficient data, these analyses will be conducted based on prespecified subgroups/covariates including age at placement, age at intervention, gender, ethnicity (indigenous vs non-indigenous), placement type (residential or group home vs foster family and kinship vs non-kinship placement), types of abuse/reason for placement (maltreatment/abuse vs behavioural problems), types of intervention (psychological, social, behavioural), duration of intervention and length of follow-up. Other factors presumed to cause variations in the outcomes may be determined during the review process, and these will be included in additional exploratory subgroup analyses.

\section{Sensitivity analysis}

Sensitivity analysis will be performed to explore the influence of heterogeneity $\left(\mathrm{I}^{2}>50 \%\right)$ and determine the robustness of the observed effect size. Specifically, the primary analysis will be repeated by altering the dataset to only include medium and high-quality studies to examine their influence on the results. If the findings are robust, then the studies of all quality will be retained, if there are 
changes in the findings, then further examination of this will be performed. Where there are sufficient numbers of studies, visual inspection of funnel plots and Begg and Egger et $a l^{29}{ }^{30}$ statistical tests will be used to assess publication bias and small study effects.

\section{Patient and public involvement}

This systematic review will not collect primary data, and therefore patients and the public were not involved in the design, conduct or reporting of the research.

\section{DISCUSSION}

Children and young people in OoHC have typically been exposed to a multitude of psychologically distressing and adverse experiences that manifests into childhood trauma. ${ }^{31}$ Childhood trauma is an important public health concern as adverse childhood experiences can have substantial health, social and economic implications which extend throughout the lifespan. ${ }^{31}$ Therefore, there is a need for health and psychosocial interventions to be implemented to prevent further traumatic and adverse childhood experiences as early as possible, as these interventions may reduce the negative outcomes of adverse childhood experiences. Existing interventions and practice models aim to directly address the impact of trauma on a child's health or psychosocial outcomes, typically through trying to reduce symptoms or facilitate recovery. ${ }^{31}$ However, many of these interventions have not been properly evaluated or have limited evidence of their effectiveness in improving the health and/or psychosocial outcomes for children and young people in OoHC. To develop effective interventions for those in OoHC, we need to understand which interventions work and how their effects can be sustained and embedded (ie, implemented) into practice. The proposed systematic review aims to address these gaps by examining how interventions and practice models can be applied to organisations and carers to improve the physical and psychosocial health of children and young people placed in OoHC. Using rigorous methodology, prespecified criteria and a predetermined search strategy, this review will capture and synthesise existing quantitative and qualitative evidence on interventions in OoHC to establish their impact in improving health and psychosocial outcomes and to disentangle the specific elements which contribute to their success. Findings from this review will provide much needed evidence to build the current knowledge base and to inform the implementation of effective interventions in $\mathrm{OoHC}$ in an effort to alleviate the poor health and psychosocial outcomes of children and young people in $\mathrm{OoHC}$.

\section{ETHICS AND DISSEMINATION}

This study does not require ethical approval as it does not involve primary data collection. We anticipate that findings from this review will contribute to an improved understanding of interventions which improve health and psychosocial outcomes for children and young people in OoHC and the key contributing factors within these interventions. These findings will be disseminated through peer-reviewed publications and at conference meetings to inform future research and to guide the development and real-world implementation of sustainable interventions in OoHC settings.

Acknowledgements EG is supported by a PhD scholarship provided by MacKillop Family Services. AM is supported by an Early Career Fellowship provided by the National Health and Medical Research Council (NHMRC) of Australia. HS is supported by a NHMRC senior research fellowship. We thank Dr Marie Misso for her input and expertise in developing the search strategy and helping to run the initial database searches.

Contributors EG developed the search strategy, wrote the first draft of the review protocol and lead the data collection and analysis. AM contributed to the design and scope of the search strategy, guided the review process and revised and edited the manuscript. RO and NH contributed to the revision and editing of the manuscript. HS determined the design and scope of the review, revised and edited the manuscript, supervised in the review process and is the guarantor for ensuring the integrity and accuracy of the review data.

Funding The authors have not declared a specific grant for this research from any funding agency in the public, commercial or not-for-profit sectors.

Competing interests None declared.

Patient consent for publication Not required.

Provenance and peer review Not commissioned; externally peer reviewed.

Open access This is an open access article distributed in accordance with the Creative Commons Attribution Non Commercial (CC BY-NC 4.0) license, which permits others to distribute, remix, adapt, build upon this work non-commercially, and license their derivative works on different terms, provided the original work is properly cited, appropriate credit is given, any changes made indicated, and the use is non-commercial. See: http://creativecommons.org/licenses/by-nc/4.0/.

\section{REFERENCES}

1. Wall L, Higgins D, Hunter C. Trauma-informed care in child/family/ welfare services. CFCA paper no 37. Melbourne: Australian Institute of Family Studies, 2016.

2. Australian Institute of Health and Welfare. Children protection Australia 2016-17. Canberra: Australian Government, 2018.

3. Jones R, Everson-Hock ES, Papaioannou D, et al. Factors associated with outcomes for looked-after children and young people: a correlates review of the literature. Child Care Health Dev 2011;37:613-22.

4. Heyes N, Smart J, Walton B, et al. Children in care. Melbourne: Australian Institute of Family Studies, 2018.

5. Leloux-Opmeer $\mathrm{H}$, Kuiper $\mathrm{C}$, Swaab $\mathrm{H}$, et al. Characteristics of children in foster care, Family-Style group care, and residential care: a scoping review. J Child Fam Stud 2016;25:2357-71.

6. Barnett ER, Boucher EA, Neubacher K, et al. Decision-Making around psychotropic medications for children in foster care: perspectives from foster parents. Child Youth Serv Rev 2016;70:206-13.

7. Meltzer H, Corbin T, Gatward R, et al. The mental health of young people looked after by local authorities in England. London: The Stationery Office, 2003.

8. Tarren-Sweeney M. The mental health of children in out-of-home care. Curr Opin Psychiatry 2008;21:345-9.

9. Tarren-Sweeney M, Hazell P. Mental health of children in foster and kinship care in New South Wales, Australia. J Paediatr Child Health 2006;42:89-97.

10. Trout AL, Hagaman J, Casey K, et al. The academic status of children and youth in out-of-home care: a review of the literature. Child Youth Serv Rev 2008;30:979-94.

11. Evans R, White J, Turley R, et al. Comparison of suicidal ideation, suicide attempt and suicide in children and young people in care and non-care populations: systematic review and meta-analysis of prevalence. Child Youth Serv Rev 2017;82:122-9. 
12. Taussig HN, Harpin SB, Maguire SA. Suicidality among preadolescent maltreated children in foster care. Child Maltreat 2014;19:17-26.

13. Trout AL, Hagaman JL, Beth Chmelka M, et al. The academic, behavioral, and mental health status of children and youth at entry to residential care. Resid Treat Child Youth 2008;25:359-74.

14. Sullivan DJ, van Zyl MA. The well-being of children in foster care: exploring physical and mental health needs. Child Youth Serv Rev 2008;30:774-86.

15. Petersen AC, Joseph J, Feit MN. New directions in child abuse and neglect research. Institute of Medicine (U.S.), National Research Council (U.S.), \& National Research Council (U.S.), 2014: 245-83.

16. McLean S, Price-Robertson R, Robinson E. Therapeutic residential care in Australia: taking stock and looking forward, 2011.

17. Bartlett JD, Rushovich B. Implementation of trauma systems Therapy-Foster care in child welfare. Child Youth Serv Rev 2018;91:30-8.

18. Vimpani GV, Webster SM, Temple-Smith MJ. Improving the health of Australian children entering out-of-home care. Med J Aust 2012;196:91-2.

19. Hill CM, Watkins J. Statutory health assessments for looked-after children: what do they achieve? Child Care Health Dev 2003;29:3-13.

20. Mooney A. Promoting the health of Looked after children: a study to inform revision of the 2002 guidance. Department for Children, Schools and Families (DCSF), 2009.

21. Barth RP, Greeson JKP, Zlotnik SR, et al. Evidence-based practice for youth in supervised out-of-home care: a framework for development, definition, and evaluation. J Evid Based Soc Work 2011;8:501-28.

22. Bailey $\mathrm{C}$, Klas A, Cox R, et al. Systematic review of organisationwide, trauma-informed care models in out-of-home care $(\mathrm{OoHC})$ settings. Health Soc Care Community 2019;27:e10-22.
23. Higgins J, Green S, eds. Cochrane Handbook for systematic reviews of interventions: the Cochrane library. Cichester, UK: John Wiley \& Sons, Ltd, 2006.

24. Apsche JA, Bass CK, Zeiter JS, et al. Family mode deactivation therapy in a residential setting: treating adolescents with conduct disorder and multi-axial diagnosis. Int $J$ Behav Consult Ther 2008;4:328-39.

25. Moher D, Shamseer L, Clarke M, et al. Preferred reporting items for systematic review and meta-analysis protocols (PRISMA-P) 2015 statement. Syst Rev 2015;4.

26. Moher D, Liberati A, Tetzlaff J, et al. Preferred reporting items for systematic reviews and meta-analyses: the PRISMA statement. PLoS Med 2009;6:e1000097.

27. Grading quality of evidence and strength of recommendations. BMJ 2004;328.

28. Higgins JPT, Thompson SG, Deeks JJ, et al. Measuring inconsistency in meta-analyses. BMJ 2003;327:557-60.

29. Begg CB, Mazumdar M. Operating characteristics of a RANK correlation test for publication bias. Biometrics 1994;50:1088-101.

30. Egger M, Davey Smith G, Schneider M, et al. Bias in meta-analysis detected by a simple, graphical test. BMJ 1997;315:629-34.

31. Gimson K, Trewhella A. Developing a Trauma-Informed therapeutic service in the Australian Capital Territory for children and young people affected by abuse and neglect. Australian Capital Territory Government: Australian Capital Territory, 2014

32. Australian Centre for Posttraumatic Mental Health and Parenting Research Centre. Approaches targeting outcomes for children exposed to trauma arising from abuse and neglect - Evidence, practice and implications, 2013. 